\title{
Unusual chemical behaviour of silylated 1,2-dithiins under Lewis acid conditions
}

\author{
Alessandro Degl'Innocenti, ${ }^{*}$ Antonella Capperucci, ${ }^{*}$ Irene Malesci and Giulio Castagnoli \\ Dipartimento di Chimica Organica, Università di Firenze, Via della Lastruccia 13,50019 Sesto Fiorentino, Italy
}

Received 9 March 2005; revised 11 May 2005; accepted 13 May 2005

\begin{abstract}
Different silylated 1,2-dithiins, obtained through self-dimerization of the related $\alpha$, $\beta$-ethylenic thioacylsilanes, in the presence of $\mathrm{AlCl}_{3}$ show an unusual rearrangement leading in good yields to novel silyl-endodisulfide bicyclic systems.

(C) 2005 Elsevier Ltd. All rights reserved.
\end{abstract}

Thiocarbonyl derivatives have gained in recent years an increasing importance in synthetic organic chemistry as useful intermediates in the synthesis of complex molecular systems and natural products. ${ }^{1}$ In this context thioacylsilanes appeared as very attractive molecules, because they couple the reactivity of the carbon-sulfur double bond with the peculiar reactivity of organosilanes, thus leading to the synthesis of various molecules containing the $\mathrm{Si}-\mathrm{C}-\mathrm{S}$ unit. $^{2}$

Very recently, our interest in the chemistry of thiocarbonyl compounds led us to uncover a mild and general access to a particular class of thioacylsilanes, namely $\alpha, \beta$-unsaturated thioacylsilanes, through our developed bis(trimethylsilyl)sulfide (HMDST) based thionation procedure, which due to its mildness proved to be useful for the synthesis of such labile derivatives. ${ }^{3-5}$

In particular, we reported that reaction of silylated allenes 1 and HMDST, in the presence of $\mathrm{CoCl}_{2} \cdot 6 \mathrm{H}_{2} \mathrm{O}$, leads to a quite convenient and general access to $\alpha, \beta$-ethylenic thioacylsilanes 2 , which undergo a head-to-head self-dimerization reaction to afford silyl-1,2-dithiins $\mathbf{3}$ as the major products in a highly regioselective way (Scheme 1). ${ }^{4}$

In the same context, transient thiopropenoylsilanes $\mathbf{2}$ were also demonstrated to be powerful 1,3-thiabutadienes being able to trap in situ generated thioaldehydes

\footnotetext{
$\overline{\text { Keywords: }}$ Unsaturated thioacylsilanes; Hetero Diels-Alder; Selfdimerization; Lewis-acid catalysis.

* Corresponding authors. Tel.: +39 055 4573551; fax: +39 055

4573585; e-mail: alessandro.deglinnocenti@unifi.it
}

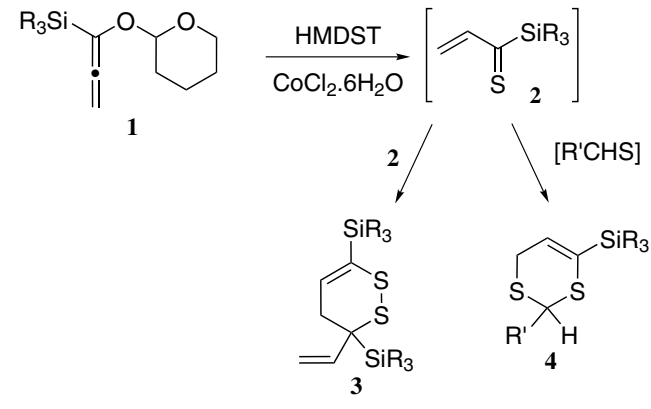

Scheme 1.

and thioacylsilanes, ${ }^{5}$ including aliphatic derivatives, leading to 2-substituted 4-silyl-1,3-dithiins 4 through a regioselective hetero Diels-Alder cycloaddition (Scheme 1). This methodology then opens a useful route to polyfunctionalized silyl 1,2- and 1,3-dithiins, which, in view of the presence of different silylated moieties, allow a possible further selective functionalization. Furthermore, the silicon moiety seems to play a fundamental role in this kind of cycloadditions. Although in fact thiopropenoylsilane can be considered a synthetic equivalent of thioacrolein, a considerable change in reactivity between such molecules can be observed. Thus, for instance, thioacrolein has never been reported to participate in cycloadditions with different thiocarbonyls, except self-dimerization. The regiochemical outcome of the self-dimerization too proved to be quite different, affording in the case of the unsaturated thioacylsilane 1,2-dithiin as the predominant adduct, instead of the 1,3-isomeric derivative, usually obtained as the major product through dimerization of thioacrolein. ${ }^{6}$ 
In this context we were then attracted by the possibility of evaluating the chemical behaviour of this kind of silylated heterocycles, and their Lewis acid promoted reactivity in the presence of dienophiles was firstly considered.

It is in fact reported that treatment with several Lewis acids (i.e., $\mathrm{AlCl}_{3}$ and $\mathrm{EtAlCl}_{2}$ ) of dithiin-type dimers 5, obtained through dimerization of $\alpha, \beta$-unsaturated aromatic thioketones, leads to regeneration of $\alpha, \beta$-ethylenic thiocarbonyls 6 that readily undergo hetero Diels-Alder reaction with different carbonyl activated dienophiles, such as for instance methyl acrylate, methyl vinyl ketone and $N, N$-dimethylacrylamide, to afford new [4+2] cycloadducts 7 (Scheme 2). ${ }^{7}$

Thus, to evaluate if the reported procedure could be applied also to the silylated 1,2-dithiins 3 , with the aim to access differently functionalized cycloadducts with carbonyl dienophiles, and also to verify whether the presence of the silyl group could somehow affect the reactivity of these silyl-heterocycles, dithiin 3a was treated at room temperature in $\mathrm{Et}_{2} \mathrm{O}$ with $\mathrm{AlCl}_{3}$ in the presence of methyl acrylate. During the analysis of the reaction mixture we came across an unexpected result, as far as we found that under these Lewis acid conditions the starting 1,2-dithiin was completely consumed, but no trace of the expected Diels-Alder cycloadduct 8 between thiopropenoylsilane and the carbonyl dienophile could be detected, while unreacted methyl acrylate was completely recovered. Nevertheless a clean reaction occurred affording a single new compound in high yield. Reaction of dithiin 3a with different activated dienophiles (i.e., methyl vinyl ketone, $N, N$-dimethylacrylamide) led invariably to the same result, thus showing the non participation of the carbonyl dienophile in the reaction. On the basis of MS, ${ }^{1} \mathrm{H}$ and ${ }^{13} \mathrm{C}$ NMR data it was possible to determine that the so obtained molecule still contained two silyl groups, but no exocyclic double bond could be evidenced, and structure 9a was suggested for this compound (Scheme 3).

X-ray analysis of crystals obtained by slow evaporation of a diethyl ether solution allowed confirmation of the proposed structure as the bicyclic sulfurated molecule 9a (Fig. 1).

The observed unusual behaviour of dithiin 3a could be ascribed to the presence of the silyl functionalities on the heterocyclic system. Aromatic thioketones $\mathbf{6}$ in fact have never been reported to undergo such a rearrangement, but only retro Diels-Alder reactions. ${ }^{7}$

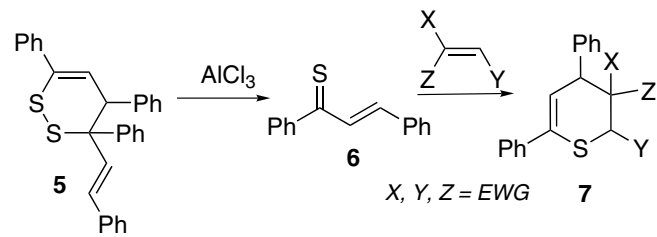

Scheme 2.

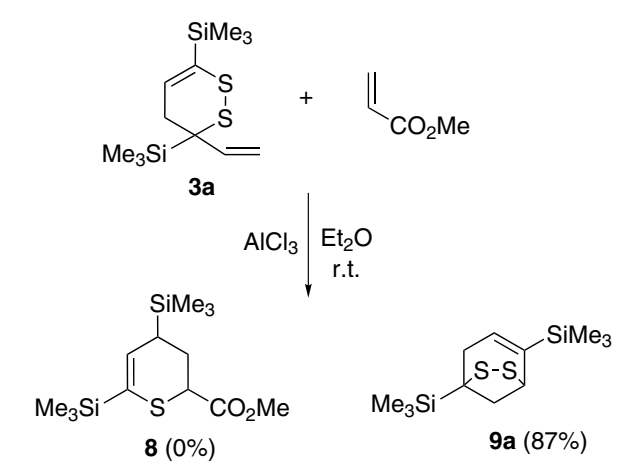

Scheme 3.

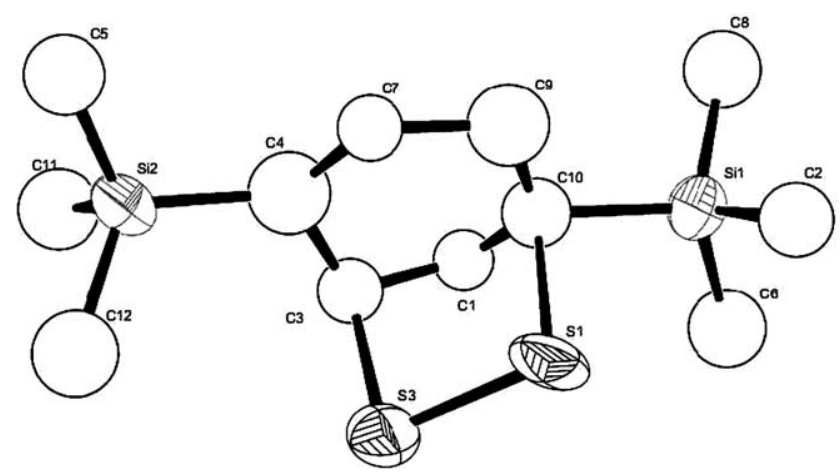

Figure 1. Molecular structure of 9a.

A tentative mechanism for the formation of compound 9a is proposed in Scheme 4: a primary attack of the Lewis acid on a sulfur atom causes the ring opening of the heterocycle. The subsequent rearrangement of the disulfide bridge, with double bond shift and hydride migration, leads to the formation of the isolated product $9 \mathbf{a}$.

It should be noted the peculiar behaviour of such silylated heterocycles, as far as it is generally observed that functionalization of structures bearing a disulfide bridge may occur through S-S bond cleavage. On the contrary, in our case, it can be envisaged a $\mathrm{C}-\mathrm{S}$ cleavage, with formation of an allylic carbocation intermediate, which, upon reaction with the vinylic carbon $\alpha$ to silicon, affords, through ring closure, a novel carbocation, stabilized by the presence of the silyl moiety on the $\beta$ position. Finally, double bond shift and hydride migration leads to compound $\mathbf{9 a}$.

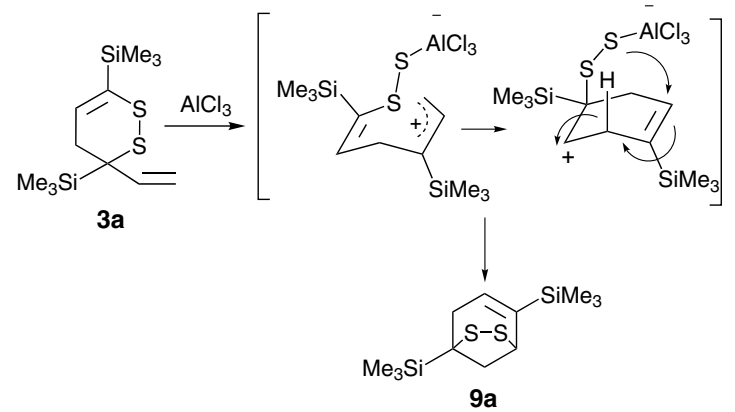

Scheme 4. 
Table 1. Treatment of 1,2-dithiins 3a-f with $\mathrm{AlCl}_{3}$

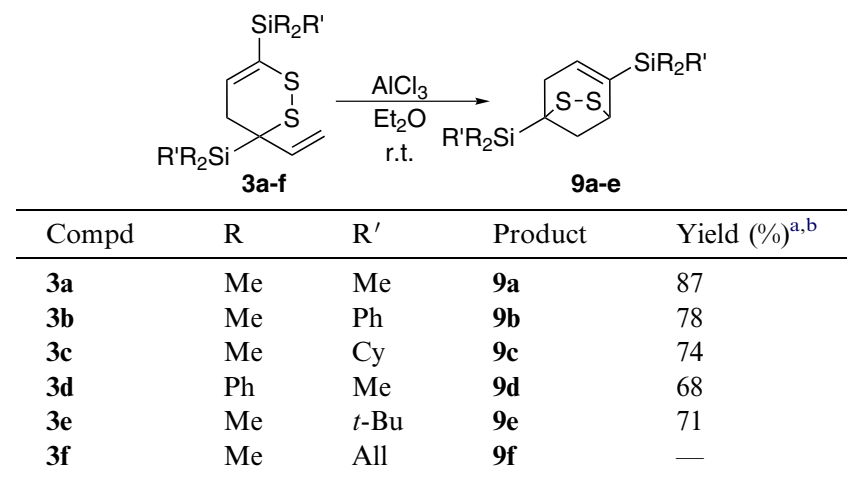

${ }^{\mathrm{a}}$ Isolated yield.

${ }^{\mathrm{b}}$ All compounds showed spectroscopical and analytical data consistent with the assigned structure.

Moreover, with the aim to evaluate whether this peculiar behaviour could be limited only to the less hindered TMS-substituted 1,2-dithiin 3a or it could configure a general one, structures bearing different groups on the silicon atom were reacted under the same conditions ${ }^{8}$ and it was observed that treatment with $\mathrm{AlCl}_{3}$ invariably led to the analogues $\mathbf{9 b}-\mathbf{e}$ of the bicyclic system obtained with the trimethylsilyl group (Table 1).

Only in the case of the $-\mathrm{SiMe}_{2} \mathrm{All}$ substituted derivative the bicyclic compound $9 f$ was not formed, probably due to a competitive reaction between the exocyclic double bond of the 1,2-dithiin $3 \mathbf{f}$ and the allyl moiety present on the silicon atom.

This new class of bicyclic structures can be considered as very interesting derivatives, as far as it is reported that structurally related molecules have been used to generate diatomic sulfur, a useful powerful dienophile to synthesize biologically active compounds, ${ }^{9}$ and similar endodisulfide systems are contained in steroids; moreover they have been described as sulfurated structural analogues of antimalarial peroxidase agents. ${ }^{10}$

Further investigations on synthetic applications of this reactivity are currently underway in our laboratory.

\section{Acknowledgements}

The authors gratefully acknowledge Professor Robert F. Cunico (Northern Illinois University, USA) for helpful discussions. Financial support by the National Project 'Stereoselezione in Sintesi Organica. Metodologie ed Applicazioni' (MURST, Roma) is gratefully acknowledged.

\section{References and notes}

1. Reviews: (a) Usov, V. A.; Timokina, L. V.; Voronkov, M. G. Sulf. Rep. 1992, 12, 95; (b) McGregor, W. M.; Sherrington, D. C. Chem. Soc. Rev. 1993, 199; (c) Metzner, P.; Thuillier, A. Sulfur Reagents in Organic Synthesis; Academic Press: London, 1994; (d) Okazaki, R. In Organosulfur Chemistry; Page, P. C. B., Ed.; Academic Press: London, 1995, Chapter 5; (e) Metzner, P. Top. Curr. Chem. 1999, 204, 127.

2. Inter alia: (a) Jones, A. D. In Comprehensive Organic Functional Group Transformations; Katritzky, A. R., Meth-Cohn, O., Rees, C. W., Eds.; Elsevier: Oxford, 1995; Vol. 5, p 647, and references cited therein; (b) Bonini, B. F.; Fochi, M. Rev. Heteroat. Chem. 1997, 16, 47, and references cited therein; (c) Bonini, B. F.; ComesFranchini, M.; Fochi, M.; Mazzanti, G.; Ricci, A.; Varchi, G. Tetrahedron Lett. 1999, 40, 6473; (d) Degl'Innocenti, A.; Capperucci, A.; Oniciu, D. C.; Katritzky, A. R. J. Org. Chem. 2000, 65, 9206.

3. Degl'Innocenti, A.; Capperucci, A. Eur. J. Org. Chem. 2000, 2171.

4. Capperucci, A.; Degl'Innocenti, A.; Biondi, S.; Nocentini, T.; Rinaudo, G. Tetrahedron Lett. 2003, 44, 2831.

5. Capperucci, A.; Degl'Innocenti, A.; Nocentini, T.; Biondi, S.; Dini, F. J. Organomet. Chem. 2003, 686, 363.

6. Li, G. M.; Niu, S.; Segi, M.; Tanaka, K.; Nakajima, T.; Zingaro, R. A.; Reibenspies, J. H.; Hall, M. B. J. Org. Chem. 2000, 65, 6601, and references cited therein.

7. Motoki, S.; Saito, T.; Karakasa, T.; Matsushita, T.; Furuno, E. J. Chem. Soc., Perkin Trans. 1 1992, 2943.

8. Typical procedure: To a solution of $24 \mathrm{mg}(0.18 \mathrm{mmol})$ of $\mathrm{AlCl}_{3}$ in $2 \mathrm{~mL}$ of anhydrous $\mathrm{Et}_{2} \mathrm{O}$ were added, under inert atmosphere, $34 \mathrm{mg}(0.12 \mathrm{mmol})$ of silylated 1,2-dithiin 3a in $1 \mathrm{ml}$ of anhydrous $\mathrm{Et}_{2} \mathrm{O}$. The mixture was stirred at $\mathrm{rt}$, following the progress of the reaction by TLC. After $18 \mathrm{~h}$, it was quenched with saturated $\mathrm{NH}_{4} \mathrm{Cl}$ and extracted with diethyl ether. The organic phase was washed with water $(3 \times 2 \mathrm{~mL})$ and brine, and dried over $\mathrm{Na}_{2} \mathrm{SO}_{4}$. Evaporation of the solvent afforded the crude product, which after purification on TLC (hexanes/Et $\mathrm{Et}_{2} \mathrm{O} 40: 1$ ) gave $29 \mathrm{mg}$ of pure compound 9a (87\%). ${ }^{1} \mathrm{H}$ NMR $\left(200 \mathrm{MHz}, \mathrm{CDCl}_{3}\right) \delta$ (ppm): $0.11(9 \mathrm{H}, \mathrm{s}), 0.16(9 \mathrm{H}, \mathrm{s}), 2.16(1 \mathrm{H}, \mathrm{ddd}$, $J=12.2 \mathrm{~Hz}, 3.4 \mathrm{~Hz}, 1.6 \mathrm{~Hz}), 2.35(1 \mathrm{H}, \mathrm{ddd}, J=18.6 \mathrm{~Hz}$, $4.4 \mathrm{~Hz}, 1.6 \mathrm{~Hz}), 2.70(1 \mathrm{H}, \mathrm{ddd}, J=18.6 \mathrm{~Hz}, J=2.2 \mathrm{~Hz}$, $J=0.8 \mathrm{~Hz}), 2.72(1 \mathrm{H}, \mathrm{dd}, J=12.2 \mathrm{~Hz}, J=1.8 \mathrm{~Hz}), 4.16$ $(1 \mathrm{H}, \mathrm{m}), 5.87(1 \mathrm{H}, \mathrm{dd}, J=4.4 \mathrm{~Hz}, J=2.2 \mathrm{~Hz}) .{ }^{13} \mathrm{C} \mathrm{NMR}$ $\left(50 \mathrm{MHz}, \mathrm{CDCl}_{3}\right) \delta(\mathrm{ppm}):-3.1,-1.7,40.0,45.5,46.1$, 50.8, 125.2, 132.8. MS m/z (\%): $288\left(\mathrm{M}^{+}, 13\right), 167$ (18), 137 (10), 135 (98), 73 (100).

9. (a) Folkins, P. L.; Harpp, D. N. J. Org. Chem. 1992, 57, 2013; (b) Gilchrist, T. L.; Wood, J. E. J. Chem. Soc., Perkin Trans. 1 1992, 9; (c) Steliou, K.; Gareau, Y.; Milot, G.; Salama, P. J. Am. Chem. Soc. 1990, 112, 7819.

10. (a) Gunatilaka, A. A. L.; Karunaratne, V.; Sandanayake, V. P.; Sotheeswaran, S. J. Chem. Res. Synop. 1990, 160; (b) Wu, Y.; Liu, H.-H. Chem. Res. Toxicol. 2003, 16, 1202, and references cited. 